\title{
Degenerate ground states and nonunique potentials: Breakdown and restoration of density functionals
}

\author{
K. Capelle, ${ }^{1}$ C. A. Ullrich, ${ }^{2}$ and G. Vignale ${ }^{2}$ \\ ${ }^{1}$ Departamento de Física e Informática, Instituto de Física de São Carlos, Universidade de São Paulo, \\ Caixa Postal 369, 13560-970 São Carlos, SP, Brazil \\ ${ }^{2}$ Department of Physics and Astronomy, University of Missouri-Columbia, Columbia, Missouri 65211, USA
}

(Received 25 February 2007; published 19 July 2007)

\begin{abstract}
The Hohenberg-Kohn (HK) theorem is one of the most fundamental theorems of quantum mechanics, and constitutes the basis for the very successful density-functional approach to inhomogeneous interacting manyparticle systems. Here we show that in formulations of density-functional theory (DFT) that employ more than one density variable, applied to systems with a degenerate ground state, there is a subtle loophole in the HK theorem, as all mappings between densities, wave functions, and potentials can break down. Two weaker theorems which we prove here, the joint-degeneracy theorem and the internal-energy theorem, restore the internal, total, and exchange-correlation energy functionals to the extent needed in applications of DFT to atoms, molecules, and solids. The joint-degeneracy theorem constrains the nature of possible degeneracies in general many-body systems.
\end{abstract}

DOI: 10.1103/PhysRevA.76.012508

\section{INTRODUCTION}

Quantum mechanics is based on the assumption that all information that one can, in principle, extract from a system in a pure state at zero temperature is contained in its wave function. In nonrelativistic quantum mechanics the wave function obeys Schrödinger's equation [1] which implies a powerful variational principle according to which the ground-state wave function minimizes the expectation value of the Hamiltonian. This variational principle was used by Hohenberg and Kohn (HK) [2] to show that the entire information contained in the wave function is also contained in the system's ground-state particle density $n(\mathbf{r})$.

HK established the existence of two mappings,

$$
v(\mathbf{r}) \stackrel{1}{\Leftrightarrow} \Psi\left(\mathbf{r}_{1}, \ldots, \mathbf{r}_{N}\right) \stackrel{2}{\Leftrightarrow} n(\mathbf{r}),
$$

where the first guarantees that the single-particle potential is a unique functional of the wave function, $v[\Psi]$, and the second implies that the ground-state wave function is a unique functional of the ground-state density, $\Psi[n]$. Taken together, both mappings are encapsulated in the single statement that the single-particle potential is a unique density functional $v[n]$. In this formulation, the HK theorem forms the basis of the spectacularly successful approach to many-body physics, electronic-structure theory, and quantum chemistry that became known as density-functional theory (DFT) [3-5].

Mapping 2 was originally proven by contradiction [2] and later by constrained search [6]. Note that, in spite of occasional statements to the contrary in the literature, neither proof directly proves the combined mapping, and thus the existence of the functional $v[n]$. This requires additionally mapping 1, which in the case of density-only DFT is proven by inverting Schrödinger's equation $[4,7]$
PACS number(s): 31.15.Ew, 03.65.-w, 71.15.Mb

$$
\hat{V}=\sum_{i} v\left(\mathbf{r}_{i}\right)=E_{k}-\frac{(\hat{T}+\hat{U}) \Psi_{k}\left(\mathbf{r}_{1}, \ldots, \mathbf{r}_{N}\right)}{\Psi_{k}\left(\mathbf{r}_{1}, \ldots, \mathbf{r}_{N}\right)} .
$$

In multidensity DFTs there is more than one density and conjugate potential, and the above inversion does not uniquely determine all potentials.

According to the preceding equation, any eigenstate $\Psi_{k}$ determines the single-particle potential $v(\mathbf{r})$ up to an additive constant, the corresponding eigenenergy [8]. The groundstate version of this equation is all one needs to prove mapping 1 , and thus of the combined mapping $n(\mathbf{r}) \Rightarrow v(\mathbf{r})$ + const, which we write as $v=v[n]$. The constant can always be absorbed in the definition of the zero of energy, but in open systems the resulting nonuniqueness of the mapping $v[n]$ gives rise to derivative discontinuities [9] that crucially contribute to observables such as the band gap, chemical hardness, electron affinities, electron transfer energies, among others.

The two classic proofs of the HK theorem, by contradiction and by constrained search, have been extended to a wide variety of systems, including spin magnetization [in spindensity-functional theory (SDFT) $[10,11]]$ and orbital currents [in current-density-functional theory (CDFT) [12]], among others. Further scrutiny, however, has led to the discovery of situations in which each of the two mappings breaks down.

\section{BREAKDOWN OF MAPPINGS: DEGENERACY}

Mapping 2, $\Psi[n]$, breaks down in the presence of degenerate ground states, where neither the constrained search proof nor the proof by contradiction provide a unique wave function among the degenerate manifold, because the strict inequality of the underlying variational principle is replaced by the weaker relation less-or-equal. In the proof by contradiction, the contradiction simply does not follow unless one has a strict inequality, and in the proof by constrained search 


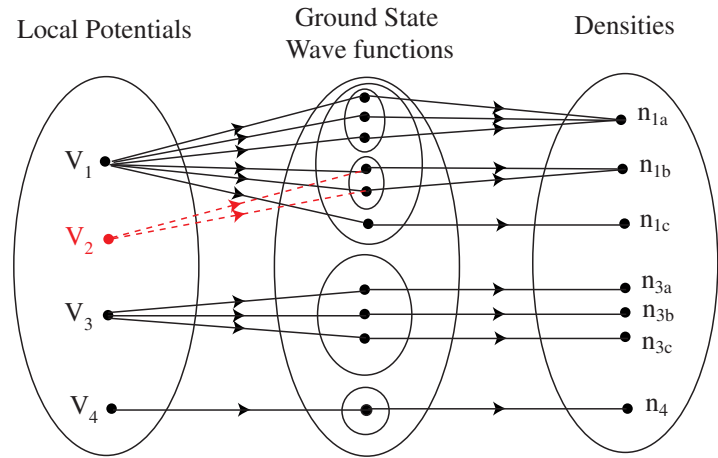

FIG. 1. (Color online) Schematic illustration of the breakdown of mappings in the presence of degeneracy (full lines) and the additional complication posed by nonuniqueness (red/dashed lines). Large ovals are sets of functions, medium-size ovals collect degenerate wave functions, and small ovals enclose degenerate wave functions that give rise to the same density. ( $v$ and $n$ are used generically to represent sets of conjugate potentials and densities.)

there is no guarantee that the search delivers only one wave function for a specified density.

What remains from the proofs is that the ground-state density uniquely determines a manifold of degenerate states, $\left\{\Psi_{i}\right\}[n]$, but not all of these states individually [13]. Any member of this manifold, however, still uniquely determines the potential, since any of them can be used in Eq. (2). Hence, even in the presence of degeneracy, the mapping $v\left[\Psi_{i}\right]$, and thus $v[n]$, still exists. This situation is illustrated in Fig. 1.

To proceed from establishing mappings to a practical density-functional theory, one must define the total-energy functional $E_{v}[n]$, the universal internal-energy functional $F[n]$, and the exchange-correlation energy functional $E_{x c}[n]$. The Kohn-Sham formulation additionally requires the noninteracting kinetic-energy functional $T_{s}[n]$. 21 years after proving the original HK theorem, Kohn [14] showed how these functionals can be defined even if degeneracy renders the original proof ineffective [4]. Since all degenerate wave functions by definition yield the same ground-state energy $E$, one can directly define the functional

$$
F[n]:=E-\int d^{3} r n(\mathbf{r}) v[n](\mathbf{r})=E-V[n] .
$$

Conventionally, this functional is defined as $F[n]=T[n]$ $+U[n]$, but the information that the kinetic energy $T$ and interaction energy $U$ are density functionals is only available if the second mapping, $\Psi[n]$, holds, and cannot be taken for granted in the presence of degeneracy. By contrast, the alternative definition above only requires the mapping $v[n]$ to establish the existence of the universal internal-energy functional $F[n]$.

Thus tamed, degeneracy actually becomes helpful in further strengthening the foundations of DFT: on a lattice, any density can be written as a linear combination of densities arising from ensembles of degenerate ground states of a local potential, thus solving the discretized $v$-representability problem [15].

\section{BREAKDOWN OF MAPPINGS: NONUNIQUENESS}

Mapping $1, v[\Psi]$, breaks down if finite basis sets are used to represent the wave functions. Harriman [16] gives both general arguments and an explicit example illustrating this breakdown, which is the only one occuring already in the charge-density-only formulation of DFT.

In multidensity DFTs, such as SDFT and CDFT, the mapping between the set of effective potentials and the set of ground-state densities can break down even in the complete basis-set limit, because inversion of Schrödinger's equation does not establish a unique relation between the set of densities and the set of conjugate potentials. This is the so-called nonuniqueness problem of SDFT (and CDFT and others). Following an early observation of the problem by von Barth and Hedin [10] the problem has been shown to be fundamental and pervasive in recent work by Eschrig and Pickett [17] and by two of the authors [18], who provided explicit examples of different SDFT potentials sharing the same ground-state wave function. Reference [18] proposed a classification of nonuniqueness into systematic (arising from the existence of certain constants of motion) and accidental (arising from special features of the ground state). In both cases, the nonuniqueness is associated with the external potential. Since the mapping $\Psi[n]$ remains intact, and internal-energy functionals can be defined exclusively in terms of wave functions,

$$
F[n]=\langle\Psi[n]|\hat{T}+\hat{U}| \Psi[n]\rangle,
$$

the functionals $E_{v}[n]=F[n]+V[n], \quad T_{s}[n]=\langle\Phi[n]|\hat{T}| \Phi[n]\rangle$, and $E_{x c}[n]=F[n]-E_{H}[n]-T_{s}[n]$ still exist. Here $\Phi$ stands for a Slater determinant, and $\Psi$ for a general many-body wave function. For notational simplicity we will in the remainder of this paper use $v$ and $n$ generically to represent sets of conjugate potentials and densities (e.g., $v_{\uparrow}, v_{\downarrow}$ and $n_{\uparrow}, n_{\downarrow}$ in collinear SDFT).

Additional examples of both systematic and accidental nonuniqueness were found in CDFT [19] and in SDFT on a lattice [20]. The extent to which nonuniqueness of the potentials affects various types of applications of multidensity DFTs, as well as possible remedies, are discussed in Refs. [19-21].

\section{BREAKDOWN OF MAPPINGS: NONUNIQUENESS AND DEGENERACY}

We have just seen that in the presence of nonuniqueness the mapping $v[\Psi]$ breaks down, whereas in the presence of degeneracy the mapping $\Psi[n]$ breaks down. Interestingly, a crucial fact has been overlooked in the standard analysis of either degeneracy or nonuniqueness: These complications can occur simultaneously. If a system with a degenerate ground state is treated with SDFT or any other formulation of DFT suffering from a nonuniqueness problem, none of the mappings hold: Degenerate ground states can produce the same density and arise from different external potentials. Under these circumstances, no conventional HK theorem exists. In fact, it is $\Psi[n]$ that is used to define $F[n]$ in the absence of 
$v[\Psi]$ (nonuniqueness), while $v[\Psi]$ guarantees the existence of $F[n]$ in the absence of $\Psi[n]$ (degeneracy). If both $\Psi[n]$ and $v[\Psi]$ break down, it seems that nothing is left. The breakdown of both mappings is illustrated in Fig. 1. Three simple examples are given below.

Our first example extends the case of the noninteracting $\mathrm{Li}$ atom [13] to collinear SDFT. If the spin degree of freedom is included, each of the four degenerate states [13] is additionally twofold degenerate with respect to $S_{z}$. The set of external potentials $B=0, v=3 / r$ thus has an eightfold degenerate ground-state manifold. The Slater determinants formed from the configurations $1 s^{2} 2 p^{+} \downarrow$ and $1 s^{2} 2 p^{-} \downarrow$ have the same charge and spin densities. Again, we see that in the presence of a degenerate ground state the densities do not uniquely determine the wave function. Differently from above, in SDFT we can now also consider the alternative set of external potentials $B^{\prime}=$ const $\neq 0, v=3 / r$. The spin-only magnetic field $B$ appears only in the Zeeman term, and simply splits the ground-state manifold into two, one comprising of the four spin-up configurations, the other of the four spin-down configurations. The new ground state will be in the spindown manifold, where the configurations $1 s^{2} 2 p^{+} \downarrow$ and $1 s^{2} 2 p^{-} \downarrow$ remain and still yield the same densities. From the point of view of the mapping between densities and potentials, this is simply the well-known [17-21] nonuniqueness of the potentials of SDFT with respect to a weak collinear magnetic field. The full situation, however, is now one in which the densities do not determine the wave functions but only a (ground-state) manifold of them, and some members of these manifolds are ground states in more than one set of external potentials. The functionals $\Psi[n], V[\Psi]$, and $V[n]$ thus do not exist.

Consider next an interacting atom in an $S=1, L=1$ state. Concrete examples are ${ }^{6} \mathrm{C}$ and ${ }^{14} \mathrm{Si}$ (with term ${ }^{3} P_{0}$ ) and ${ }^{8} \mathrm{O}$ and ${ }^{16} \mathrm{~S}$ (with term ${ }^{3} P_{2}$ ). In the set of external potentials $B$ $=0, v=Z / r$ the ground state of such systems is $(2 L+1)(2 S$ $+1)=$ ninefold degenerate. Let us denote the members of this manifold as $\Psi_{L_{z}, S_{z}}$. Several of these, such as $\Psi_{1,1}$ and $\Psi_{-1,1}$ have the same charge and spin densities. Hence, we have another situation in which these densities do not determine the wave functions but only the manifold. Now consider the same system in external potentials $B^{\prime}=$ const $\neq 0$ and $v$ $=Z / r$. The states $\Psi_{1,1}, \Psi_{0,1}$, and $\Psi_{-1,1}$ remain degenerate ground states in this new set of potentials, and the density and spin density of the first and the last are still the same as for $B^{\prime}=0$. Hence, as in other examples of nonuniqueness, knowledge of this state alone does not determine the external potentials. Upon combining both observations we find that to a given set of ground-state densities $(n, m)$ there may correspond more than one degenerate wave function (all in local external potentials $B=0, v=Z / r$ ), and all of these wave functions are also degenerate ground states of the different set of local external potentials $\left(B^{\prime}, v\right)$. Again, the functionals $\Psi[n]$, $V[\Psi]$, and $V[n]$ do not exist.

Last, we discuss a modification of the one-electron example by von Barth and Hedin [10]. Consider a single electron in the presence of an external four-potential $w_{\alpha \beta}(\mathbf{r})$ $=V(\mathbf{r}) \delta_{\alpha \beta}-[\mathbf{B}(\mathbf{r}) \cdot \vec{\sigma}]_{\alpha \beta}$, where $\vec{\sigma}$ is the vector of Pauli matrices. Let $w_{\alpha \beta}(\mathbf{r})$ be uniform along one spatial direction (say,
(I)

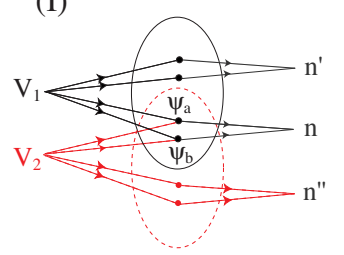

(II)

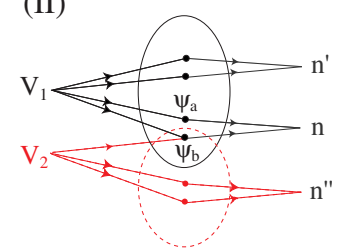

FIG. 2. (Color online) The joint-degeneracy theorem states that two degenerate ground states with equal density in a potential $V_{1}$ can also be ground states of a second potential $V_{2}$, but only as a degenerate pair. Case II, in which one of the two states is a ground state of $V_{2}$ but the other is not, is excluded by the theorem.

$x$ ), with periodic boundary conditions along that direction separated by a distance $L$ (which is topologically equivalent to confining the electron on a ring). The twofold degenerate ground state of the Hamiltonian $H_{\alpha \beta}=-\frac{\hbar^{2}}{2 m} \delta_{\alpha \beta}+w_{\alpha \beta}(\mathbf{r})$ is given by $\Psi_{\alpha}^{ \pm}(\mathbf{r})=e^{ \pm i k x} \psi_{\alpha}(y, z), k=2 \pi / L$, with both ground states producing the same density. Furthermore, both ground states $\Psi_{\alpha}^{ \pm}(\mathbf{r})$ are invariant under perturbations $w_{\alpha \beta}^{\prime}(\mathbf{r})$ $=V^{\prime}(\mathbf{r})\left\{\delta_{\alpha \beta}-[\mathbf{m}(\mathbf{r}) \cdot \vec{\sigma} / n(\mathbf{r})]_{\alpha \beta}\right\}$, where $n(\mathbf{r})$ and $\mathbf{m}(\mathbf{r})$ are the ground-state density and magnetization, and $V^{\prime}(\mathbf{r})$ is an arbitrary (but not too large, in order to avoid level crossings [18]) scalar potential function. Thus, we have found another case where both mappings (1) break down.

\section{RESTORATION OF ENERGY FUNCTIONALS}

The question then arises whether the energy functionals $E_{v}[n], F[n], T_{s}[n]$, and $E_{x c}[n]$ can still be defined, even in the absence of all mappings that are conventionally considered in the content of the HK theorem. To answer affirmatively, we consider two distinct cases, represented in Fig. 2. In case I, $\Psi_{a}$ and $\Psi_{b}$, which both produce density $n$, are degenerate ground states in potentials $V_{1}$ and $V_{2}$. In case II only $\Psi_{b}$ is a common ground state of both potentials, whereas $\Psi_{a}$ is a ground state only of $V_{1}$, but either an excited state or not even an eigenstate at all in $V_{2}$. We first prove that case II cannot occur. In potential $V_{1}$, we define the internal energy (not yet a functional of any density) as

$$
F_{1}=E_{1}-\int d^{3} r v_{1}(\mathbf{r}) n(\mathbf{r}) .
$$

In potential $V_{1}, \Psi_{a}$ and $\Psi_{b}$ are degenerate, so that $E_{1}$ $=\left\langle\Psi_{a}\left|\hat{T}+\hat{U}+\hat{V}_{1}\right| \Psi_{a}\right\rangle=\left\langle\Psi_{b}\left|\hat{T}+\hat{U}+\hat{V}_{1}\right| \Psi_{b}\right\rangle$. Since both also produce the same density, the expectation value of $\hat{V}_{1}$ with $\Psi_{a}$ and $\Psi_{b}$ is the same, so that

$$
\left\langle\Psi_{a}|\hat{T}+\hat{U}| \Psi_{a}\right\rangle=\left\langle\Psi_{b}|\hat{T}+\hat{U}| \Psi_{b}\right\rangle=F_{1}
$$

is independent of the choice of wave function. Next, applying the variational principle to the Hamiltonian of system 2, we have

$$
\left\langle\Psi_{a}\left|\hat{T}+\hat{U}+\hat{V}_{2}\right| \Psi_{a}\right\rangle>E_{2},
$$

with a strict inequality, as by assumption $\Psi_{a}$ in potential 2 is not degenerate with the ground state of that system. From 
Eq. (6), and again making use of the fact that $\Psi_{a}$ and $\Psi_{b}$ produce the same density, we can write this as

$$
\left\langle\Psi_{b}\left|\hat{T}+\hat{U}+\hat{V}_{2}\right| \Psi_{b}\right\rangle=E_{2}>E_{2} .
$$

The contradiction proves that case II cannot occur. This result is completely general, and implies the following theorem: Consider two degenerate ground-state wave functions in potential $V_{1}, \Psi_{a}$, and $\Psi_{b}$. The constraint that these two wave functions have the same density guarantees that in any other potential $V_{2}$ either both are ground states (and thus also degenerate) or none of them is. We call this the jointdegeneracy theorem. Note that all of our explicit examples above respect the joint-degeneracy theorem. Here, we use this theorem to prove the existence of the energy functionals of DFT even in situations in which all three standard mappings, $\Psi[n], V[n]$, and $V[\Psi]$, break down. However, we stress that the theorem is valid beyond DFT, and quite generally constrains the nature of possible degeneracies in many-body systems.

Even though one could formally define an $F[n]$ functional in case II, we have just shown that this case cannot occur, so we only need to establish the existence of $F[n]$ in case I. In analogy to Eq. (5), we define in potential $V_{2}$,

$$
F_{2}=E_{2}-\int d^{3} r v_{2}(\mathbf{r}) n(\mathbf{r}) .
$$

Since $\Psi_{a}$ and $\Psi_{b}$ are degenerate also in potential $V_{2}$, we have $E_{2}=\left\langle\Psi_{a}\left|\hat{T}+\hat{U}+\hat{V}_{2}\right| \Psi_{a}\right\rangle=\left\langle\Psi_{b}\left|\hat{T}+\hat{U}+\hat{V}_{2}\right| \Psi_{b}\right\rangle$, resulting in

$$
F_{2}=\left\langle\Psi_{a}|\hat{T}+\hat{U}| \Psi_{a}\right\rangle=\left\langle\Psi_{b}|\hat{T}+\hat{U}| \Psi_{b}\right\rangle,
$$

where we again used that $\Psi_{a}$ and $\Psi_{b}$ yield the same density. This is the same equation obtained above for $F_{1}$. Again, this result is completely general, implying the following theorem: Regardless of any possible degeneracy or nonuniqueness, two systems with the same ground-state density have the same internal energy $F$. Hence, the functional $F[n]$ exists and is universal, i.e., independent of the potentials. This internal- energy theorem is consistent with the constrained-search formulation of DFT [6], which defines $F[n]:=\min _{\Psi \rightarrow n}\langle\Psi| \hat{T}$ $+\hat{U}|\Psi\rangle$, although in the presence of degeneracy this definition cannot be used to define $\Psi[n]$.

Since the noninteracting kinetic energy $T_{s}$ is the internal energy of the Kohn-Sham system, it is also a well-defined density functional, and $E_{x c}[n]=F[n]-E_{H}[n]-T_{s}[n]$ can be constructed as usual. Finally, for a given external potential, the functional $E_{v}[n]$ then obviously also exists.

\section{CONCLUSIONS}

We have shown both by general arguments and by specific examples that in the case of degeneracy in multidensity DFTs all three mappings, $\Psi[n], V[\psi]$, and $V[n]$, and thus the entire body of information usually considered in the content of the HK theorem, break down. The weaker jointdegeneracy and internal-energy theorems, however, still allow the definition of the internal-energy functional $F[n]$, and thus also of the functionals $T_{s}[n], E_{v}[n]$, and $E_{x c}[n]$. However, we stress that we have only proven existence of the functionals, not their differentiability. In fact, in open systems all these functionals are expected to display derivative discontinuities. Ensemble DFT is the proper framework for addressing these questions, but we note that multidensity ensemble DFT itself is subject to the same kind of questions we here ask for multidensity pure-state DFT. In particular, uniqueness of the external potentials cannot be taken for granted.

\section{ACKNOWLEDGMENTS}

One of the authors (K.C.) was supported by FAPESP and CNPq. One of the authors (C.A.U.) acknowledges support from DOE Grant No. DE-FG02-05ER46213, NSF Grant No. DMR-0553485, and Research Corporation. One of the authors (G.V.) acknowledges support from NSF Grant No. DMR-0313681.
[1] E. Schrödinger, Ann. Phys. 79, 361 (1926).

[2] P. Hohenberg and W. Kohn, Phys. Rev. 136, B864 (1964).

[3] W. Kohn, Rev. Mod. Phys. 71, 1253 (1999).

[4] R. M. Dreizler and E. K. U. Gross, Density Functional Theory (Springer-Verlag, Berlin, 1990).

[5] R. G. Parr and W. Yang, Density-Functional Theory of Atoms and Molecules (Oxford University Press, Oxford, 1989).

[6] M. Levy, Phys. Rev. A 26, 1200 (1982); E. H. Lieb, in Density Functional Methods in Physics, edited by R. M. Dreizler and J. da Providencia (Plenum, New York, 1985).

[7] M. Levy, Adv. Quantum Chem. 21, 69 (1990).

[8] The subscript $k$ labels the entire spectrum of the system. The ground-state wave function $\Psi_{k=0}$ in this paper is simply denoted by $\Psi$. If there is more than one degenerate ground-state wave function these are labelled $\Psi_{i}$.

[9] L. J. Sham and M. Schlüter, Phys. Rev. Lett. 51, 1888 (1983);
J. P. Perdew and M. Levy, ibid. 51, 1884 (1983); J. P. Perdew, R. G. Parr, M. Levy, and J. L. Balduz, ibid. 49, 1691 (1982).

[10] U. von Barth and L. Hedin, J. Phys. C 5, 1629 (1972).

[11] O. Gunnarsson and B. Lundqvist, Phys. Rev. B 13, 4274 (1976).

[12] G. Vignale and M. Rasolt, Phys. Rev. Lett. 59, 2360 (1987); Phys. Rev. B 37, 10685 (1988).

[13] A simple example [4,22] is the noninteracting Li atom with the four degenerate ground-state configurations $1 s^{2} 2 s, 1 s^{2} 2 p^{+}$, $1 s^{2} 2 p^{0}$, and $1 s^{2} 2 p^{-}$. Slater determinants formed from the second and fourth configuration are distinct wave functions that have the same density $n(\mathbf{r})$ and arise from the same external potential.

[14] W. Kohn, in Highlights of Condensed Matter Theory, edited by F. Bassani, F. Funi, and M. P. Tosi (North-Holland, Amsterdam, 1985). 
[15] J. T. Chayes, L. Chayes, and M. B. Ruskai, J. Stat. Phys. 38, 497 (1985); C. A. Ullrich and W. Kohn, Phys. Rev. Lett. 89, 156401 (2002); 87, 093001 (2001); P. E. Lammert, J. Chem. Phys. 125, 074114 (2006).

[16] J. E. Harriman, Phys. Rev. A 27, 632 (1983).

[17] H. Eschrig and W. E. Pickett, Solid State Commun. 118, 123 (2001).

[18] K. Capelle and G. Vignale, Phys. Rev. Lett. 86, 5546 (2001).

[19] K. Capelle and G. Vignale, Phys. Rev. B 65, 113106 (2002).
[20] C. A. Ullrich, Phys. Rev. B 72, 073102 (2005).

[21] N. Gidopolous, Phys. Rev. B 75, 134408 (2007); N. Argaman and G. Makov, Phys. Rev. B 66, 052413 (2002); O. Gritsenko and E. J. Baerends, J. Chem. Phys. 120, 8364 (2004); W. Kohn, A. Savin, and C. A. Ullrich, Int. J. Quantum Chem. 100, 20 (2004); P. W. Ayers and W. Yang, J. Chem. Phys. 124, 224108 (2006).

[22] R. M. Martin, Electronic Structure (Cambridge University Press, Cambridge, 2004). 